\title{
Disordered dislocation configuration in submicrometer Al crystal subjected to plane strain bending
}

\author{
T. Hu, K. Ma, T.D. Topping, L. Jiang, D. Zhang, A.K. Mukherjee, J.M. Schoenung, E.J. Lavernia* \\ Department of Chemical Engineering \& Materials Science, University of California, Davis, CA 95616, \\ USA \\ *Corresponding author email: Enrique J. Lavernia, lavernia@ucdavis.edu
}

\begin{abstract}
Bending tests of submicrometer Al pillars were performed in-situ in a transmission electron microscope (TEM). The Al crystal in the bent region experienced substantial lattice distortion, as well as grain refinement resulting in a disordered dislocation configuration arising from a random distribution of low angle grain boundaries (LAGBs). This observed disordered dislocation configuration is in contrast to formation of a low energy dislocation configuration, as predicted on the basis of the theory of strain gradient plasticity, for bulk materials subjected to plane strain bending.
\end{abstract}

Keywords:in-situ TEM; submicrometerpillars; strain bending; dislocation configuration; low angle grain boundary 
There is a vast body of literature, published primarily during the last decade, documenting size dependent phenomena in crystals when sample dimensions are reduced to the order of micrometers and/or submicrometers $[1,2]$. For example, novel deformation mechanisms in submicrometer and micrometer crystals, e.g., dislocation starvation and single arm dislocation sources, have been proposed and verified via both theory and experimentation [3-9]. Research addressing size dependent phenomena has been motivated not only by interesting fundamental observations, but also by the ever increasing trend to miniaturize devices, which frequently involve micropillars, nanowires, thin films and submicron interconnections, for applications in micro- and nanoelectromechanical systems (MEMS, NEMS) [10-12].

Ultimately, the successful design of micrometer- or submicrometer-sized devices will require careful evaluation of mechanical properties, as well as establishing a fundamental understanding of microstructure evolution at these length scales. Review of the published literature reveals that, in most studies, the mechanical properties of materials with micrometric or nanometric scales have been established using uniaxial tension and/or compression testing [7,13-15]. A notable exception involves MEMS/NEMS systems, and in some of these studies the structural crystals were subjected to non-uniaxial loading conditions, such as bending and twisting, which resulted in plasticity gradients during deformation $[11,16,17]$. It is known that when a crystal is deformed, dislocations are initially generated, followed by movement (e.g., glide and/or climb) and accumulation (e.g., storage). Moreover, dislocations are generally stored in response to: 1) accumulation as dislocations interact and immobilize each other in a random way, and/or 2) the need to maintain continuity during deformation. In the former case the dislocations are referred to as statistically stored dislocations (SSDs), whereas the latter ones are described as geometrically necessary dislocations (GNDs) and are related to gradients during plastic deformation. The shear strength of a material can be simply expressed by Taylor's relation: $\tau=$ $\alpha \mu b \sqrt{\rho_{S}+\rho_{G}}$, where $\rho_{S}$ is the density of SSDs and $\rho_{G}$ is the density of GNDs [18]. According to Fleck and Hutchinson's theory of strain gradient plasticity, both SSDs and GNDs have the most efficient dislocation configurations, which determine the work hardening response of a deformed material [19]. It 
then follows that an understanding of how a dislocation configuration evolves is a prerequisite to properly describe strain gradient plasticity. In related studies, a few in-situ bending tests conducted have been reported in an effort to establish deformation mechanism and ascertain plastic stability [10,20,21]. However, inspection of the published literature shows that studies of dislocation configurations in crystals subjected to bending has heretofore never been reported. Motivated by this lack of information, we conducted in-situ bending tests of submicron Al crystals in a transmission electron microscope (TEM). Our results show that the Al crystals experienced a significant extent of polygonization during bending. Moreover, we documented the formation of highly disordered dislocation configurations during bending, in apparent contradiction to the low energy configurations predicted on the basis of Fleck and Hutchinson's theory [19].

The material used in this study is a precipitation strengthened $7075 \mathrm{Al}$ alloy. The bulk material was fabricated via cryomilling and consolidation and has an ultrafine grained (UFG) microstructure [22]. The as-received material was subjected to $\mathrm{T} 6$ heat treatment (solutionized at $480^{\circ} \mathrm{C}$ for 1 hour, quenched in ice water and then annealed at $120^{\circ} \mathrm{C}$ for 24 hours). Then the bulk material was ground and polished down to a thickness of $\sim 40 \mu \mathrm{m}$. The jet-polishing was subsequently applied to acquire a free-standing thin foil with a thickness of 1-2 $\mu \mathrm{m}$. This thin foil was used to section submicrometer pillars by using a FEI Quanta 3D FEG dual beam SEM/FIB system. The pillars with square cross-section have a side length of $\sim 150 \mathrm{~nm}$ and a height of $\sim 1340 \mathrm{~nm}$ as shown in Fig. 1(a). The aspect ratio (ratio between length and the side length) of this pillar is about 9, which ensures the occurrence of bending in the middle section of the pillar. The average tapering angle of the pillar is $\sim 3^{\circ}$, which is an accepted value in many studies [3,5,13,20,23]. In-situ bending tests were performed using a Hysitron PI95 picoindenter (Hysitron, MN, USA) equipped with a flat tip in a JEOL 2500 (JEOL, Tokyo, Japan) TEM operated at $200 \mathrm{kV}$ (see supplementary material). After the in-situ bending test, the bent region was further thinned by FIB to perform postmortem TEM characterization. $5 \mathrm{kV}$ and $77 \mathrm{pA}$ were applied to final thinning to minimize the surface defects caused by the ion beam. There is no occurrence of ion-beam induced bulking or deflection 
[24], which potentially affects the microstructure. More details about the materials and in-situ bending test can be found in supporting material.

Prior to the in-situ bending test, the submicrometer Al pillar was documented to contain several grains with sizes ranging from $100 \mathrm{~nm}$ to $460 \mathrm{~nm}$, as shown in Fig. 1(a). In Fig. 1(b), the SAED pattern was taken from the grain, as circled in Fig. 1(a). The sharp diffraction spots indicate that this single grain is close to the [011] zone axis and that it was not subjected to lattice distortion. Fig. 1(c) shows the morphology of the pillar after the bending test. The SAED pattern in Fig. 1(d) was taken from the bent region, as circled in Fig. 1(c), which was the same single grain as indicated in Fig. 1(a). Distinctive diffraction rings were observed in the diffraction pattern. Careful examination shows that, despite the presence of discrete diffraction spots, the rings were mainly composed of curved and elongated streaks. It is known that each diffraction spot corresponds to the reflection from a specific lattice plane. When the spot is elongated, it indicates that the lattice is subject to substantial distortion [25]. In Fig. 1(d), the elongated streaks in the SAED pattern indicate that the bent region was subject to substantial lattice distortion. The inset dark field TEM image in Fig. 1(c) also confirms that there is no prominent grain refinement.Instead the refined grains were dramatically elongated and exhibited a rippled morphology.

To reveal the detailed microstructure of the bent region, the deformed pillar was further thinned by FIB, and postmortem HRTEM characterization was conducted. Fig. 2(a) shows the presence of three elongated grains, which are highlighted in the bent region. By tilting $9^{\circ}$ within the TEM, several more elongated grains with similar morphologies emerged, as shown in Fig. 2(b). It can be seen that these elongated grains exhibit irregular morphologies. Moreover, the orientation of these elongated grains is random. Some of grains are bent and conformed to the contour of the bent pillar, while others intersected with the contour.A high magnification view of the elongated grains consisting of multiple segments, with an average length of $\sim 23 \mathrm{~nm}$ and a width of $\sim 10 \mathrm{~nm}$ is shown in Fig. 2(c). What appears to be the relative displacement between adjacent segments causes the stair-like profile of the elongated grain. This geometry is likely attributed to an overall sliding between adjacent segments as this region accommodates 
the strain of the entire grain. The interface between two segments was further characterized by HRTEM. As shown in Fig. 2(d) and (e), there is an array of edge dislocations aligned at the interface, which is characteristic of a low angle grain boundary (LAGB) [26]. This indicates that the elongated grain contains multiple segments connected by LAGBs. As the LAGBs are randomly distributed in the bent region of the submicrometer pillar, the dislocations piled up along the LAGBs are therefore randomly distributed throughout the entire strained region of the pillar.

During bending, as shown schematically in Fig. 3(a), a plastically deformed crystal at the macroscale or micrometer scale contains GNDs that lie along active slip planes in the manner suggested in Fig. 3(b) and (c). In Fig. 3(b), the dislocations are randomly distributed on the active slip planes. The edge dislocations with the same sign accumulate on the same slip planes, which results in an additive strain field, but with a random distribution on the slip planes [25]. To attain a minimum strain energy and a minimum density of GNDs, a different configuration of the same dislocations is shown in Fig. 3(c). This configuration for plane strain bending has been described as the most efficient dislocation configuration on the basis of Fleck and Hutchinson's theory of strain gradient plasticity [19]. In this configuration, the GNDs are organized into arrays aligned in a direction normal to the slip planes. These GNDs in arrays thus constitute the LAGBs. However, in the case of the submicrometer-sized pillar studied herein, it is found that the LAGBs and dislocations are randomly distributed throughout the entire bent region. The dislocation configuration is illustrated in Fig. 3(d). For a short range, the GNDs align along the LAGBs, which are the interfaces between segments, as shown in Fig. 2(b) and Fig. 2(c). However, the distribution of GNDs exhibits a disordered arrangement over the long range, which is substantially different from the scenario of plane strain bending in a bulk crystal.

In a macroscopic specimen, the number of slip planes and the spatial size of the slip planes are significantly larger than those present in the submicrometer pillar. Therefore, dislocations can glide easily and remain on their slip planes in macroscopic samples. We ascribed the unusual dislocation structure in submicrometer pillars to three reasons: (1) the occurrence of grain polygonization (shown in Fig. 1c and 
d), which broke down the continuity of the slip bands that a high density of dislocations involved. Rotation of the refined grainsalteredthe direction of the slip planes. Consequently, LAGBs will not evolve along the radial direction, which in turn changed the dislocation configuration. It is noted that, the polygonization in the deformed pillar was observed without annealing. It is thus argued that dislocation glide was the dominant mechanism at the beginning. However, the dislocation climb occurred to promote the dislocation arrays (forming low angle grain boundaries)even without sufficient thermal activated process; (2) fast escape of SSDs in submicrometer pillars. The actual value of dislocation density in a bent strip is approximately $15.6 \times 10^{12} \mathrm{~m}^{-2}$ [27]. According to Nye and Ashby's equation $\rho_{\mathrm{G}}=\mathrm{k}^{\mathrm{P}} / \mathrm{b}_{1}$, where $\mathrm{k}^{\mathrm{P}}$ is the plastic curvature and $b_{1}$ is the component of the Burgers vector, the density of GNDsis about $9.7 \times$ $10^{12} \mathrm{~m}^{-2}$ in a bent strip with an effective height of $3.57 \mu \mathrm{m}[28,29]$. This means that nearly one third of the dislocations in the bent strip are "statistical" rather than geometrically necessary. These SSDs will stochastically escape from the free surface due to the sample dimension effect [1-4] and result in a configuration with randomly distributed dislocations; (3) in this case the precipitates willpin dislocations and exacerbatethe unusual dislocation configuration.

In summary, the bent region of the submicrometer pillar has some similarities to the bulk crystal after bending, which are described as follows. First, the bent region is subjected to substantial lattice distortion. Second, GNDs were aligned to form LAGBs to minimize the strain energy. However, the dislocation configuration in the bent region of the submicrometer pillar is distinctive compared to the dislocation configuration in bulk samples, as predicted on the basis of the theory by Fleck and Hutchinson. The configuration of GNDs is disordered in the bent region due to the randomly distributed LAGBs in the elongated grains.

This work was supported by the Office of Naval Research under contract no. ONR N00014-12-10237 with Dr. L. Kabacoff as program officer, and Defense University Research Instrumentation Program (DURIP) under grant no. ONR N00014-110788. The FIB milling was performed at the Laboratory for Electron and X-ray Instrumentation (LEXI) at the University of California, Irvine, using instrumentation 
funded in part by the National Science Foundation Center for Chemistry at the Space-Time Limit (CHE082913).

[1] M.D. Uchic, D.M. Dimiduk, J.N. Florando, W.D. Nix, Science 305 (2004) 986-989

[2] J.R. Greer, J.Th.M. De Hosson, Prog. Mater. Sci. 56 (2011) 654-724

[3] Z. Shan, R.K. Mishra, S.A. Syed Asif, O.L. Warren, A. Minor, Nat. Mater. 7 (2008) 115-119

[4] S.H. Oh, M. Legros, D. Kiener, G. Dehm, Nat. Mater. 8 (2009) 95-100

[5] J.R. Greer, W.D. Nix, Phys. Rev. B 73 (2006) 245410

[6] S.I. Rao, D.M. Dimiduk, T.A. Parthasarathy, M.D. Uchic, M. Tang, C. Woodward, Acta Mater. 56 (2008) 3245-3259

[7] D. Jang, J.R. Greer, Scripta Mater. 64 (2011) 77-80

[8] S. Xu, Y.F. Guo, A.H.W. Ngan, Int. J. Plasticity 43 (2013) 116-127

[9] X.W. Gu, C.N. Loynachan, Z. Wu, Y.W. Zhang, D.J. Srolovitz, J.R. Greer, Nano Lett. 12 (2012) 6385-6392

[10] M. Legros, D.S. Gianola, C. Motz, MRS Bulletin 35 (2010) 354-360

[11] W.D. Nix, J.R. Greer, G. Feng, E.T. Lilleodden, Thin Solid Films 515 (2007) 3152-3157

[12] K. Zheng, X. Han, L. Wang, Y. Zhang, Y. Yue, Y. Qin, X. Zhang, Z. Zhang, Nano Lett. 9 (2009) 2471-247

[13] D. Kiener, W. Grosinger, G. Dehm, R. Pippan, Acta Mater. 56 (2008) 580-592

[14] A.T. Jennings, J.R. Greer, Philos. Mag. 91 (2010) 1-13

[15] K.S. Ng, A.H.W. Ngan, Philos. Mag. 89 (2009) 3013-3026

[16] C. Wang, Y. Mao, Z. Shan, M. Dao, J. Li, J. Sun, E. Ma, S. Suresh, Proc. Natl. Acad. Sci. U.S.A. 110 (2013) 19725-19730

[17] Z. Shan, JOM, 64 (2012) 1229-1234

[18] H. Gao, Y. Huang, W.D. Nix, J.W. Hutchinson, J. Mech. Phys. Solids 47 (1999) 1239-1263

[19] N.A. Fleck, G.M. Muller, M.F. Ashiby, J.W. Hutchinson, Acta Metall. Mater. 42 (1994) 475-487

[20] C. Chen, Y. Pei, J.T.M. De Hosson., Acta Mater. 58 (2010) 189-200

[21] L. Wang, P. Liu, P. Guan, M. Yang, J. Sun, Y. Cheng, A. Hirata, Z. Zhang, E. Ma, M. Chen, X. Han, Nat. Commun. 4 (2013) 2413 
[22] T. Hu, K. Ma, T.D. Topping, J.M. Schoenung, E.J. Lavernia, Acta Mater. 61 (2013) 2163-2178

[23] D. Kiener, A.M. Minor, Acta Mater. 59 (2011) 1328-1337

[24] S. Punzhin, E. Detsi, A. Kuzmin, Jeff D. Hosson, J. Mater. Sci. 49 (2014)5598-5605

[25] R.E. Reed-Hill, R. Abbaschian, Physical metallurgy principles, PWS-Kent Publishing Company, Boston, US 1992

[26] W.T. Read, W. Shockley, Physical Review 78 (1950) 275-289

[27] H.H.M. Cleveringa, E. Van der Giessen, A. Needleman, Int. J. Plasticity 15 (1999) 837-868

[28] J.F. Nye, Acta. Metall. 1 (1953) 153-162

[29] M.F. Ashby, Phil. Mag. 21 (1970) 399-424 
Graphic Abstract:Bending tests of submicrometer Al pillars were performed in-situ in a transmission electron microscope (TEM). The unique findings are: 1) the Al crystal in the bent region experienced substantial lattice distortion, as well as grain refinement; 2) a disordered dislocation configuration arising from a random distribution of low angle grain boundaries (LAGBs). This observed disordered dislocation configuration is in contrast to the prediction on the basis of the theory of strain gradient plasticity, for bulk materials subjected to plane strain bending.

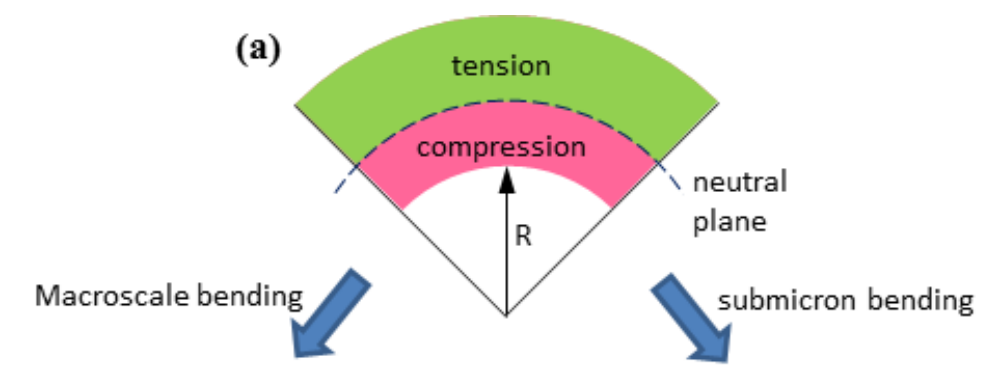

\section{(b)}
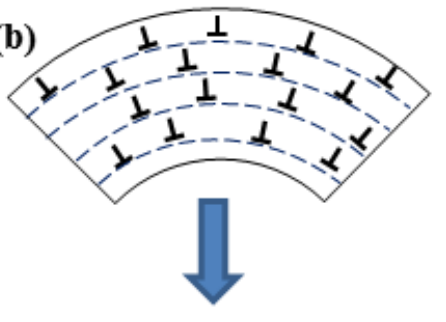

(c) (d)

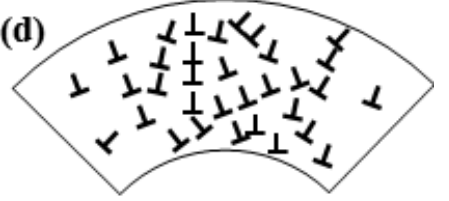

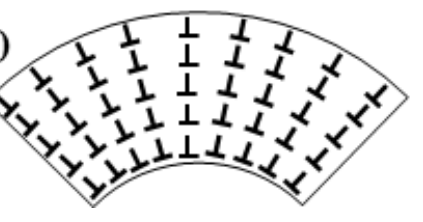




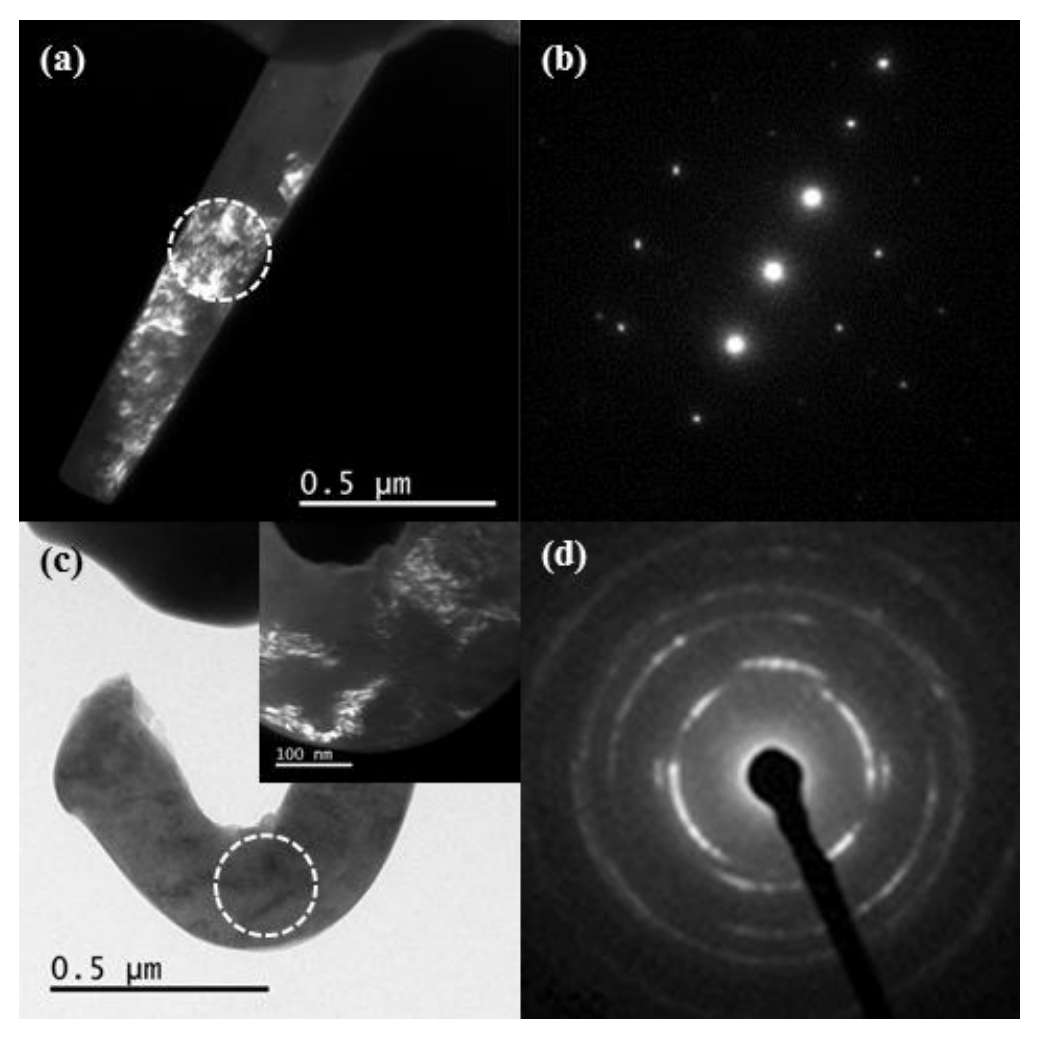

Fig. 1 


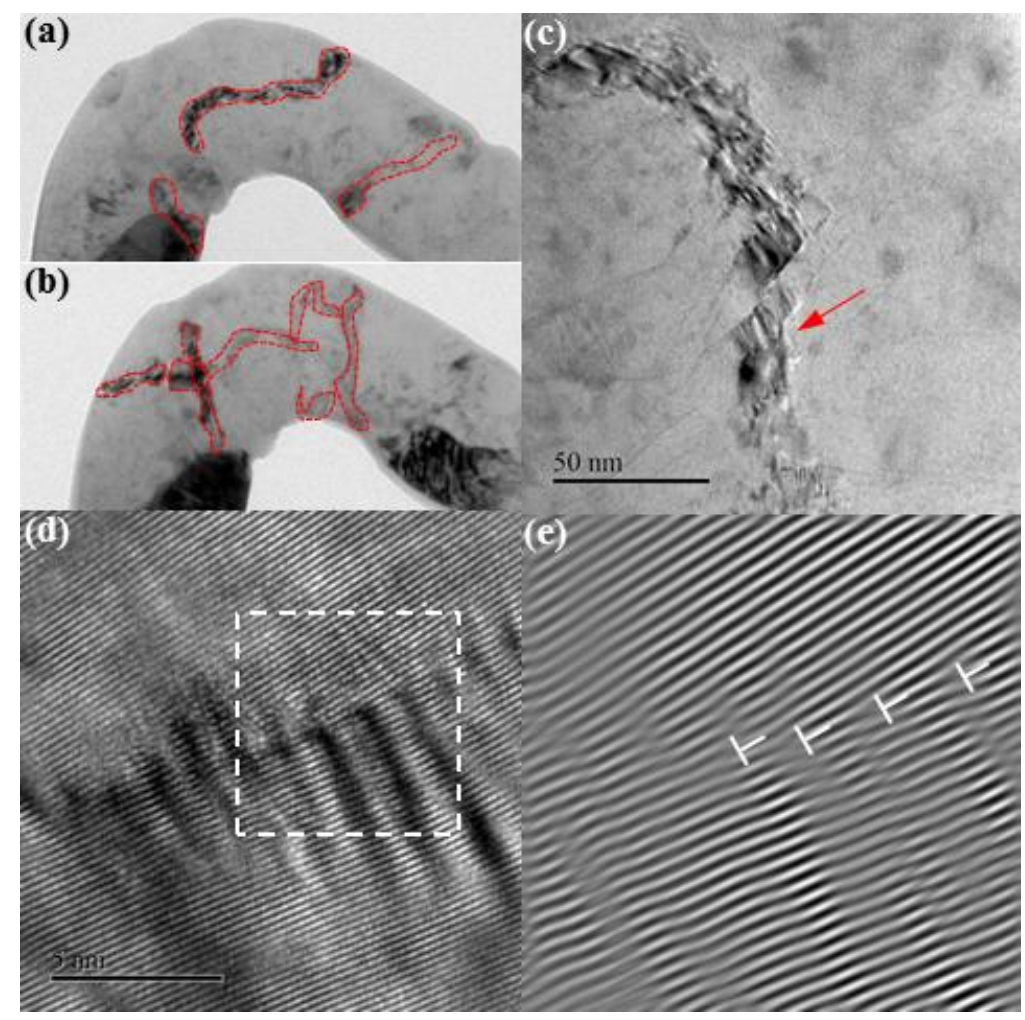

Fig. 2 


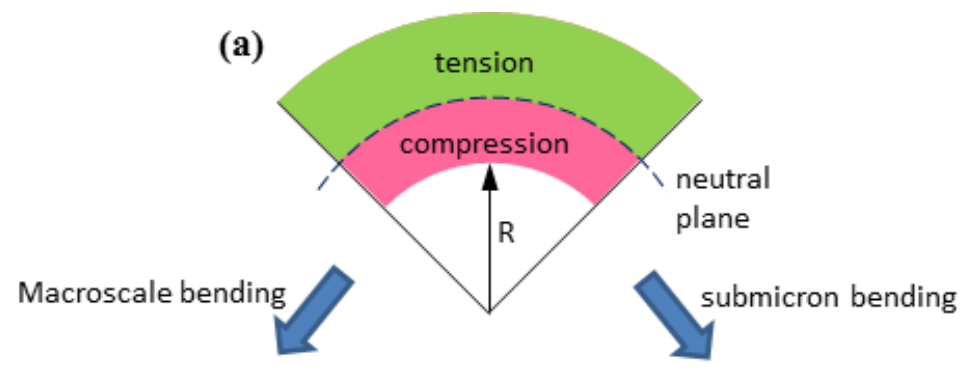

(b)

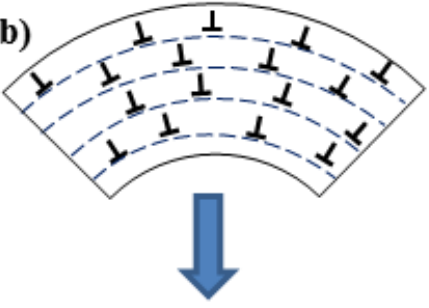

(c)

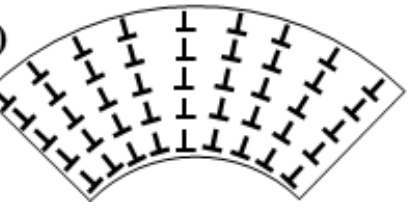

(d)

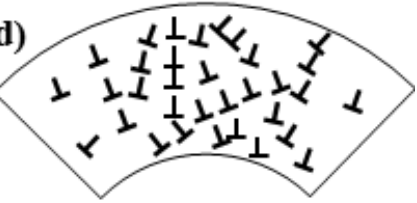

Fig. 3 